Serum glucose level normalized 20 hours after treatment started. The diagnosis of T1DM was made based on elevated blood antibodies against islet cells (ICA) and islet antigen 2 (IA-2) antibodies. He was discharged after 10 days without any complications related to HHS and is being regularly followed up. Since HHS has high mortality rate, early recognition and proper management are necessary for a better outcome.

\section{NEUROENDOCRINE TUMOURS OF THE APPENDIX}

Melša Matea*, Krnić Nevena, Rojnić Putarek Nataša, Špehar Uroić Anita, Pavlović Maja, Bilić Ernest. Klinika za pedijatriju, KBC Zagreb

\subsection{6/archdischild-2021-europaediatrics.218}

Introduction Neuroendocrine tumours (NETs) are rare tumours in paediatric population. They are most often located at appendix and are usually diagnosed accidentally, as a result of pathohistological evaluation after appendectomy, following acute appendicitis. They are found in $0.3 \%$ appendectomies in children, more often in girls (56\%), with median age of 12 years at the time of diagnosis. The prognosis is usually favourable and depends on the size and location of the tumour, protrusion into the mesoappendix, metastatic potential (Ki-67 index) and possible local metastasis. NET of the appendix can rarely be found as a part of some genetic diseases, such as multiple endocrine neoplasia (MEN) type 1 and 2, neurofibromatosis, tuberous sclerosis and von Hippel-Lindau disease. Due to the small number of individuals affected, there are no standardised diagnostic and therapeutic guidelines in paediatric population.

Case Report Here we report two cases of boys in whom pathohistological evaluation following appendectomies performed due to acute appendicitis revealed appendiceal NETs in both patients. The appendectomy upon the first patient was performed at 12.4 years of age. The tumour was located at the apex of the appendix, it was up to $0.5 \mathrm{~cm}$ in diameter, well differentiated, with low mitotic index (Ki-67 around 1\%) and with clear resection margins. Family history revealed that the boy's grandfather was treated for appendiceal tumour at the age of 17 years. The other patient was operated at 12.8 years of age. The tumour was located at the apex of the appendix, in its muscular layer, with the length of $0.6 \mathrm{~cm}$ and Ki67 4\% - grade 2 NET. During the follow-up (6 years and 2 months) neither relapse nor other tumours occurred. The additional diagnostic procedure revealed elevated parathormone levels (7.7 ..7.3 ..7.46 pmol/L, norm. 1.0-6.0 pmol/L), while blood calcium levels were at the upper limit of normal range (up to $2.57 \mathrm{mmol} / \mathrm{L}$, norm. up to $2.53 \mathrm{mmol} / \mathrm{L}$ ), blood phosphate levels were normal, vitamin $\mathrm{D}$ levels periodically lowered $(60 \ldots 51 \mathrm{nmol} / \mathrm{L}$, norm. $>75 \mathrm{nmol} / \mathrm{L})$, despite the peroral substitution therapy, and the US examination of the neck was normal. Molecular genetic analysis did not reveal mutations characteristic for multiple endocrine neoplasia type 1 .

Discussion Neuroendocrine tumours of the appendix have an excellent prognosis, particularly tumours smaller than $1 \mathrm{~cm}$, with low mitotic index, located in the apex of the appendix and with clear resection margins. When such cases occur (as described here in two of our patients), appendectomy is considered a therapeutic method of choice. Our patients did not experience a relapse of the tumour. Furthermore, there were no signs of associated diseases during the follow-up (6.2 years and 1 year), which is consistent with the data published in literature (a 100\% survival rate and no evidence of relapse occurrence). Even though aforementioned tumours are described for the most part in girls, both of our patients are boys. Reports on patients with this rare form of appendiceal tumour will contribute to accumulation of data regarding the course and prognosis of the disease and will contribute to development of guidelines for treatment and monitoring of paediatric patients, which are currently insufficient.

\section{ADRENAL SUPPRESSION WITH GROWTH RETARDATION IN AN INFANT TREATED WITH TOPICAL OCULAR GLUCOCORTICOIDS}

Nataša Rojnic Putarek*, Nevena Krnic, Sanda Huljev Frkovic, Gordana Colig, Milivoj Jovancevic. Poliklinika Arista, Zagreb, School of Medicine 'Juraj Dobrila' University of Pula, School of Medicine University of Zagreb

\subsection{6/archdischild-2021-europaediatrics.219}

Introduction Corticosteroid therapy can lead to severe iatrogenic adrenal insufficiency (IAI) and Cushing syndrome. These side-effects are common with systematic corticosteroid treatment and are less common with topical treatment modalities, even though it has been described with corticosteroid inhalation, trans- or intradermal use, intranasal and intraarticular corticosteroid application. Although ocular administration was thought to rarely lead to the development of IAI, recent studies have shown that more than two-thirds of children develop some degree of adrenal suppression, and patients who have developed an adrenal crisis during anaesthesia have been described.

Case Report We present a female infant who developed postoperative glaucoma following the surgery for congenital cataract. The therapy with topical corticosteroid eye preparations was started at the age of 5.5 months [Maxitrol drops (dexamethasone 0,1\%), Tobradex ointment (dexamethasone $1 \mathrm{mg}$ ) $\mathrm{g})$, at an average dose of $1.5 \mathrm{mg} /$ day dexamethasone. At the age of 8.7 months, 3 months after the initiation of corticosteroid use, she was examined by a paediatric endocrinologist for growth retardation [BW $6190 \mathrm{~g}(<3 \mathrm{ct},-2.1$ SDS), BH $63 \mathrm{~cm}$ $(<3$ ct, -2.4 SDS)]. There were no other clinical signs of Cushing syndrome or other growth hormone deficiency associated syndromes. Laboratory finding revealed low ACTH levels with suppressed cortisol secretion (ACTH 1,1 pmol/L; norm 1,6-13,9; cortisol $<3 \mathrm{nmol} / \mathrm{lL}$, norm 138-690). Further investigation ruled out other endocrine causes of reduced growth and congenital malformations of other organ systems. A normal female karyotype was found by classical karyotyping, 46, XX. Analysis of a panel of genes whose mutations are associated with congenital cataracts found no pathogenic variants. The girl was of normal intellectual development, and the result of pharmacogenetic analysis showed normal metabolism of corticosteroid drugs. Despite the recommendation to gradually reduce the dose of corticosteroids, local therapy was abruptly discontinued and at the age of 9 months the girl was hospitalized due to the development of an adrenal crisis. Hydrocortisone replacement therapy was initiated $(10 \mathrm{mg} / \mathrm{m} 2 /$ day) and topical ocular corticosteroids (dexamethasone 0,1\%) were continued in the dose of 2-5 drops daily. Adrenal function recovered after 10 months (ACTH short test $250 \mu \mathrm{g} / \mathrm{m} 2$; cortisol 60' $523,7 \mathrm{nmol} / \mathrm{L})$, followed by growth spurt. At the age of 3 years and 4 months, the girl reached 25-50 centile in high-for-age curve (-0.15 SDS) which is in accordance with 